2009; 00:1-19

\title{
Robust multigrid preconditioners for the high-contrast biharmonic plate equation ${ }^{\dagger}$
}

\author{
Burak Aksoylu ${ }^{1 *}$, Zuhal Yeter ${ }^{1}$ \\ ${ }^{1}$ Department of Mathematics $\&$ Center for Computation and Technology, Louisiana State University
}

N

\author{
SUMMARY
}

We study the high-contrast biharmonic plate equation with HCT and Morley discretizations. We construct a preconditioner that is robust with respect to contrast size and mesh size simultaneously based on the preconditioner proposed by Aksoylu et al. (2008, Comput. Vis. Sci. 11, pp. 319-331). By extending the devised singular perturbation analysis from linear finite element discretization to the above discretizations, we prove and numerically demonstrate the robustness of the preconditioner. Therefore, we accomplish a desirable preconditioning design goal by using the same family of preconditioners to solve elliptic family of PDEs with varying discretizations. We also present a strategy on how to generalize the proposed preconditioner to cover high-contrast elliptic PDEs of order $2 k, k>2$. Moreover, we prove a fundamental qualitative property of solution of the high-contrast biharmonic plate equation. Namely, the solution over the highly-bending island becomes a linear polynomial asymptotically. The effectiveness of our preconditioner is largely due to the integration of this qualitative understanding of the underlying PDE into its construction.

KEY WORDS: Biharmonic equation, plate equation, fourth order elliptic PDE, Schur complement, lowrank perturbation, singular perturbation analysis, high-contrast coefficients, discontinuous coefficients, heterogeneity.

\section{INTRODUCTION}

We study the construction of robust preconditioners for the high-contrast biharmonic plate equation (also referred as the biharmonic equation). The aim is to achieve robustness with respect to the contrast size and the mesh size simultaneously, which we call as $m$ - and $h$ robustness, respectively. In the case of a high-contrast diffusion equation, we studied the family of preconditioners $B_{A G K S}$ by proving and numerically demonstrating that the same family used for finite element discretization [4] can also be used for conservative finite volume discretizations with minimal modification [6]. In this article, we extend the applicability of $B_{A G K S}$ even further and show that the very same preconditioner can be used for a wider

${ }^{*}$ Correspondence to: Burak Aksoylu: Center for Computation and Technology, Louisiana State University, 216 Johnston Hall, Baton Rouge LA, 70803 USA

$\dagger$ Email: burak@cct.lsu.edu 
family of elliptic PDEs. The broadness of the applicability of $B_{A G K S}$ has been achieved by singular perturbation analysis (SPA) as it provides valuable insight into qualitative nature of the underlying PDE and its discretizations. In order to study the robustness of $B_{A G K S}$, we use an SPA that is similar to the one devised on the matrix entries by Aksoylu et al. [4]. SPA turned out to be an effective tool in analyzing certain behaviors of the discretization matrix $K(m)$ such as the asymptotic rank, decoupling, low-rank perturbations (LRP) of the resulting submatrices. LRPs are exploited to accomplish dramatic computational savings and this is the main numerical linear algebra implication.

The devised SPA is utilized to explain the properties of the submatrices related to $K(m)$. In particular, SPA of highly-bending block $K_{H H}(m)$, as modulus of bending $m \rightarrow \infty$, has important implications for the behaviour of the Schur complement $S(m)$ of $K_{H H}(m)$ in $K(m)$. Namely,

$$
S(m):=K_{L L}-K_{L H} K_{H H}^{-1}(m) K_{H L}=S_{\infty}+\mathcal{O}\left(m^{-1}\right),
$$

where $S_{\infty}$ is a LRP of $K_{L L}$. The rank of the perturbation depends on the number of disconnected components comprising the highly-bending region. This special limiting form of $S(m)$ allows us to build a robust approximation of $S(m)^{-1}$ by merely using solvers for $K_{L L}$ by the help of the Sherman-Morrison-Woodbury formula.

Preconditioning for the biharmonic equation was extensively studied in the domain decomposition setting $[23,31]$ and multigrid, BPX, and hierarchical basis settings $[8,14,22,17$, $26,27]$. Other solution strategies were also developed such as fast Poisson solvers [20, 21] and iterative methods [11]. However, there is only limited preconditioning literature available for discontinuous coefficients. Marcinkowski [19] studied domain decomposition preconditioners for the mortar type discretization of the biharmonic equation with large jumps in the coefficients.

The high-contrast in material properties is ubiquitous in composite materials. Hence, the modeling of composite materials is an immediate application of the biharmonic plate equation with high-contrast coefficients. Since the usage of composite materials is steadily increasing, the simulation and modeling of composite has become essential. We witness that the utilization of composites has become an industry standard. For instance, light weight composite materials are now being used in modern aircrafts by Airbus and Boeing. There is imminent need for robust preconditioning technology in the computational material science community as the modeling and simulation capability of composites evolve.

In [29], the Euler-Bernoulli equation with discontinuous coefficients was studied for the kinematics of composite beams. In the beam setting, the physical meaning of the PDE coefficient corresponds to the product of Young's modulus and moment of inertia [28] [p. 103], [29]. In the biharmonic plate equation setting, the PDE coefficient represents the plate modulus of bending [28] [p. 406]. Nonhomogeneous elastic plates has been considered in [18] with varying modulus of elasticity.

Our model problem is limited to the biharmonic equation which captures only the isotropic materials. The extension of our analysis to a more generalized 4-th order PDE is widely open. Such PDEs have an important role in structural mechanics as they are used in modeling anisotropic materials. Plane deformations of anisotropic materials were studied in [24], but extension to simultaneously heterogeneous and anisotropic case needs to be further explored. Grossi [13] has studied the existence of the weak solutions of anisotropic plates. The coercivity of the bilinear forms has also been established which may lay the foundations for our future work related to LRPs.

2009; 00:1-19 
The remainder of the article is structured as follows. In $\S 2$, we present the underlying highcontrast biharmonic plate equation and the associated bilinear forms. Subsequently, the effects of high-contrast on the spectrum of stiffness matrix and its subblocks are also discussed. Since the proposed preconditioner is based on LRP, in $\S 3$, we study the LRP of the limiting Schur complement as in (1.1). In $\S 4$, we present the aforementioned SPA and reveal the asymptotic qualitative nature of the solution. In particular, the solution over the highly-bending region converges to a linear polynomial as $m \rightarrow \infty$. In $\S 5$, we introduce the proposed preconditioner and prove its effectiveness by establishing a spectral bound for the preconditioned system. In $\S 6$, a strategy is presented on how to generalize the proposed preconditioner to cover highcontrast elliptic PDEs of order $2 k, k>2$. In $\S 7$, the $m$ - and $h$-robustness of the preconditioner are demonstrated by numerical experiments.

\section{THE UNDERLYING PDE AND THE LINEAR SYSTEM}

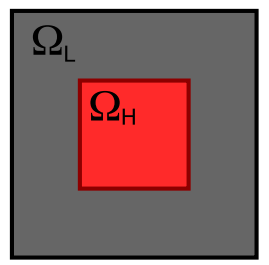

Figure 1. $\Omega=\bar{\Omega}_{H} \cup \Omega_{L}$ where $\Omega_{H}$ and $\Omega_{L}$ are highly- and lowly-bending regions, respectively.

We study the following high-contrast biharmonic equation for the clamped plate problem:

$$
\begin{array}{rll}
\nabla^{2}\left(\alpha \nabla^{2} u\right)=f & \text { in } \Omega \subset \mathbb{R}^{2}, \\
u=\partial_{n} u=0 & \text { on } \partial \Omega .
\end{array}
$$

We restrict the plate bending process to a binary regime (see Figure 1) in which the coefficient $\alpha$ is a piecewise constant function with the following values:

$$
\alpha(x)= \begin{cases}m \gg 1, & x \in \Omega_{H}, \\ 1, & x \in \Omega_{L} .\end{cases}
$$

It is quite common to idealize the discontinuous PDE coefficient $\alpha$ by a piecewise constant function $[7,16]$. In the case of high-contrast diffusion equation, Aksoylu and Beyer [1] showed that the idealization of diffusivity by piecewise constant coefficients is meaningful by showing a continuous dependence of the solutions on the diffusivity; also see [2]. A similar justification can be extended to the high-contrast biharmonic plate equation.

\subsection{Bilinear forms for the biharmonic equation}

In the theory of elasticity, potential energy is defined by using rotationally invariant functions. For plates, the potential energy is given by $[9$, p. 30$]$ :

$$
J(v):=\frac{1}{2} \int_{\Omega} \alpha\left[\{\text { trace Hess }\}^{2}+2(\sigma-1) \operatorname{det} H e s s\right] d x-\int_{\Omega} f v d x,
$$

2009; 00:1-19 
where Hess is the Hessian,

$$
H e s s=\left[\begin{array}{ll}
\partial_{11} v & \partial_{12} v \\
\partial_{21} v & \partial_{22} v
\end{array}\right]
$$

The bilinear form corresponding to energy minimization in (2.2) is given by:

$$
a(u, v):=\int_{\Omega} \alpha\left[\nabla^{2} u \nabla^{2} v+(1-\sigma)\left\{2 \partial_{12} u \partial_{12} v-\partial_{11} u \partial_{22} v-\partial_{22} u \partial_{11} v\right\}\right] d x,
$$

where $0<\sigma<1 / 2$ is the Poisson's ratio. Note that the straightforward bilinear form associated to (2.1) is obtained by using Green's formula:

$$
\int_{\Omega} \nabla^{2}\left(\alpha \nabla^{2} u\right) v d x=\int_{\Omega} \alpha \nabla^{2} u \nabla^{2} v d x+\int_{\partial \Omega} \alpha \partial_{n} \nabla^{2} u v d \gamma-\int_{\partial \Omega} \alpha \nabla^{2} u \partial_{n} v d \gamma
$$

We see that both (2.3) and (2.4) contain the so-called canonical bilinear form, $\tilde{a}(u, v)$, associated to the biharmonic equation (2.1):

$$
\tilde{a}(u, v):=\int_{\Omega} \alpha \nabla^{2} u \nabla^{2} v d x .
$$

When $u, v \in H_{0}^{2}(\Omega)$, both bilinear forms $a(u, v)$ and $\tilde{a}(u, v)$ correspond to the strong formulation (2.1) due to second Green's formula and the zero contribution of the below term:

$$
\int_{\Omega}(1-\sigma)\left\{2 \partial_{12} u \partial_{12} v-\partial_{11} u \partial_{22} v-\partial_{22} u \partial_{11} v\right\} d x
$$

\subsection{Effects of high-contrast on the spectrum}
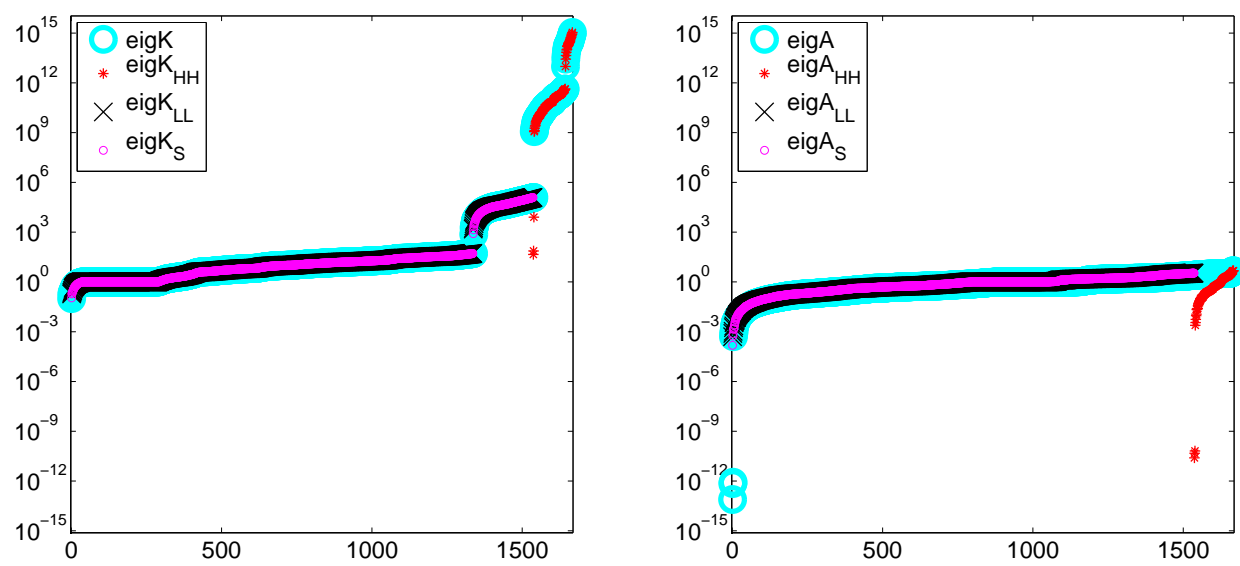

Figure 2. The HCT discretization of the biharmonic equation with $m=10^{10}$. (Left) The spectrum of the stiffness matrix $K$. (Right) Spectrum of the diagonally scaled stiffness matrix. Notice the 3 small eigenvalues of order $\mathcal{O}\left(\mathrm{m}^{-1}\right)$ corresponding to the kernel of the Neumann matrix, $\operatorname{span}\left\{\underline{1}_{H}, \underline{x}_{H}, \underline{y}_{H}\right\}$. The plot of the two of smallest eigenvalues overlap because they are roughly of the same magnitude. 
Roughness of PDE coefficients causes loss of robustness of preconditioners. This is mainly due to clusters of eigenvalues with varying magnitude. Although diagonal scaling has no effect on the asymptotic behaviour of the condition number, it leads to an improved clustering in the spectrum. The spectrum of diagonally scaled stiffness matrix, $A$, is bounded from above and below except three eigenvalues in the case of a single isolated highly-bending island. On the other hand, the spectrum of $K$ contains eigenvalues approaching infinity with cardinality depending on the number of DOF contained within highly-bending island. For the case of HCT discretization with $m=10^{10}$, we depict the spectra of $K$ and $A$ and their subblocks in Figure 2. Clustering provided by diagonal scaling can be advantageous for faster convergence of Krylov subspace solvers especially when deflation methods designed for small eigenvalues are used; for further discussion see [5].

Utilizing the matrix entry based analysis by Graham and Hagger [12] for linear FE, in [6], the authors extended the spectral analysis to cell-centered FV discretization and obtained an identical spectral result for $A$. Namely, the number of small eigenvalues of $A$ depends on the number of isolated islands comprising the highly-bending region. We observe a similar behaviour for the biharmonic plate equation where the only difference is that for each island we observe three small eigenvalues rather than one. The three dimensional kernel of the Neumann matrix is responsible for that difference; see $\S 3$. A similar matrix entry based analysis can be applied to discretizations of the plate equation, but this analysis is more involved for HCT and Morley discretizations than that for linear FE. Hence, we exclude it from scope of this article.

\section{DISCRETIZATIONS AND LOW-RANK PERTURBATIONS}

We consider an $H^{2}$-conformal and also an $H^{2}$-nonconformal Galerkin finite element discretization; Hsieh-Clough-Tocher (HCT) [10] and Morley [25] elements, respectively. Let the linear system arising from the discretization be denoted by:

$$
K(m) x=b .
$$

$\Omega$ is decomposed with respect to magnitude of the coefficient value as

$$
\Omega=\bar{\Omega}_{H} \cup \Omega_{L},
$$

where $\Omega_{H}$ and $\Omega_{L}$ denote the highly- and lowly-bending regions, respectively. DOF that lie on the interface, $\Gamma:=\bar{\Omega}_{H} \cap \bar{\Omega}_{L}$, between the two regions are included in $\Omega_{H}$. When $m$-dependence is explicitly stated and the discretization system (3.1) is decomposed with respect to (3.2), i.e., the magnitude of the coefficient values, we arrive at the following $2 \times 2$ block system:

$$
\left[\begin{array}{ll}
K_{H H}(m) & K_{H L} \\
K_{L H} & K_{L L}
\end{array}\right]\left[\begin{array}{l}
x_{H} \\
x_{L}
\end{array}\right]=\left[\begin{array}{l}
b_{H} \\
b_{L}
\end{array}\right]
$$

There are important properties associated to the $K_{H H}$ block in (3.3): It is the only block that has $m$-dependence, and furthermore, a matrix with low-rank kernel can be extracted from it. Our preconditioner construction is based on LRPs from this extraction. Next, we explain how to extract the so-called Neumann matrix and why $a(u, v)$ is the suitable bilinear form for that purpose.

By rewriting (2.3) as the following

$$
a(u, v)=\int_{\Omega} \alpha\left[\sigma \nabla^{2} u \nabla^{2} v+(1-\sigma)\left\{\partial_{11} u \partial_{11} v+\partial_{22} u \partial_{22} v+2 \partial_{12} u \partial_{12} v\right\}\right] d x
$$

2009; 00:1-19 
we see that

$$
\begin{aligned}
a(v, v) & =\alpha \sigma\left\|\nabla^{2} v\right\|_{L_{2}(\Omega)}^{2}+\alpha(1-\sigma)|v|_{H^{2}(\Omega)}^{2} \\
& \geq \alpha(1-\sigma)|v|_{H^{2}(\Omega)}^{2} .
\end{aligned}
$$

The inequality (3.5) has important implications. Namely, $a(v, v)$ is $V_{\mathcal{P}_{1}}(\Omega)$-coercive where $V_{\mathcal{P}_{1}}(\Omega) \subset H^{2}(\Omega)$ is a closed subspace such that $V_{\mathcal{P}_{1}}(\Omega) \cap \mathcal{P}_{1}=\emptyset$ and $\mathcal{P}_{1}$ denotes the set of polynomials of degree at most 1. Furthermore, (3.5) immediately implies that $a(v, v)$ is $H_{0}^{2}(\Omega)$-coercive.

Let $\mathcal{T}^{h}$ be the triangulation of $\Omega$. Based on $\mathcal{T}^{h}$, we define the associated discrete space $V_{\mathcal{P}_{1}}^{h}(\Omega)$ such that $V_{\mathcal{P}_{1}}^{h} \cap \mathcal{P}_{1}^{h}=\emptyset$. A precise definition of the $K_{H H}$ block in the stiffness matrix in (3.1) is given by:

$$
\left\langle K_{H H} \underline{\phi}_{H}^{h}, \underline{\psi}_{H}^{h}\right\rangle:=a\left(\phi_{H}^{h}, \psi_{H}^{h}\right),
$$

where $\phi_{H}^{h}, \psi_{H}^{h} \in V^{h}\left(\Omega_{H}\right) \subset H_{0}^{2}\left(\Omega_{H}\right)$ are the basis functions. We define the Neumann matrix $\mathcal{N}_{H H}$ as follows:

$$
\left\langle\mathcal{N}_{H H} \underline{\phi}_{H}, \underline{\psi}_{H}\right\rangle:=a\left(\phi_{H}^{h}, \psi_{H}^{h}\right),
$$

where $\phi_{H}^{h}, \psi_{H}^{h} \in V_{\mathcal{P}_{1}}^{h}\left(\Omega_{H}\right)$. Since $a(\cdot, \cdot)$ is $V_{\mathcal{P}_{1}}(\Omega)$-coercive, this implies by $(3.5)$ that

$$
\operatorname{ker} \mathcal{N}_{H H}=\left.\mathcal{P}_{1}^{h}\right|_{\bar{\Omega}_{H}}=\operatorname{span}\left\{\underline{1}_{H}, \underline{x}_{H}, \underline{y}_{H}\right\} .
$$

Hence, $K_{H H}(m)$ has the following decomposition:

$$
K_{H H}(m)=m \mathcal{N}_{H H}+R
$$

where $R$ is the coupling matrix corresponding to DOF on the interface $\Gamma$. Now, we are in a position to reveal the resulting main numerical linear algebra implication. As $m \rightarrow \infty$, the limiting Schur complement $S_{\infty}$ in (1.1) becomes a rank-3 perturbation of $K_{L L}$. This result relies on the fact that the inverse of the limiting $K_{H H}$ is of rank-3; see (4.1). This is due to the fact that $\mathcal{N}_{H H}$ has a rank 3 kernel whose (normalized) discretization is given by:

$$
e_{H}:=\left[\underline{1}_{H}, \underline{x}_{H}, \underline{y}_{H}\right]
$$

\section{MAIN SINGULAR PERTURBATION ANALYSIS RESULTS}

Lemma 4.1. The asymptotic behaviour of the submatrices in (5.1) is given by the following:

$$
\begin{aligned}
K_{H H}(m)^{-1} & =e_{H} \eta^{-1} e_{H}^{t}+\mathcal{O}\left(m^{-1}\right), \\
S(m) & =K_{L L}-\left(K_{L L} e_{H}\right) \eta^{-1}\left(e_{H}^{t} K_{L L}\right)+\mathcal{O}\left(m^{-1}\right), \\
K_{L H} K_{H H}(m)^{-1} & =\left(K_{L L} e_{H}\right) \eta^{-1} e_{H}^{t}+\mathcal{O}\left(m^{-1}\right),
\end{aligned}
$$

where

$$
\eta:=e_{H}^{t} K_{H H} e_{H}
$$

Proof Since $\mathcal{N}_{H H}$ is symmetric positive semidefinite, using (3.6) we have the following spectral decomposition where $n_{H}$ denotes the cardinality of DOF in $\bar{\Omega}_{H}$ :

$$
Z^{t} \mathcal{N}_{H H} Z=\operatorname{diag}\left(\lambda_{1}, \ldots, \lambda_{n_{H}-3}, 0,0,0\right)
$$


where $\left\{\lambda_{i}: i=1, \ldots, n_{H}\right\}$ is a non-increasing sequence of eigenvalues of $\mathcal{N}_{H H}$ and $Z$ is orthogonal. Since, the eigenvectors corresponding to the zero eigenvalues are discretization of the polynomials $1, x$, and $y$, we can write $Z=\left[\tilde{Z} \mid e_{H}\right]$ where $e_{H}$ is defined in (3.8). Using (3.7), we have:

$$
\begin{aligned}
Z^{t} K_{H H}(m) Z & =\left[\begin{array}{cc}
m \operatorname{diag}\left(\lambda_{1}, \ldots, \lambda_{n_{H}-3}\right)+\tilde{Z}^{t} R \tilde{Z} & \tilde{Z}^{t} R e_{H} \\
& e_{H}^{t} R \tilde{Z}
\end{array}\right. \\
& =:\left[\begin{array}{cc}
\tilde{\Lambda}(m) & \tilde{\delta} \\
\tilde{\delta}^{t} & \eta
\end{array}\right] .
\end{aligned}
$$

To find the limiting form of $K_{H H}(m)^{-1}$ note that

$$
\begin{aligned}
\tilde{\Lambda}(m) & =m \operatorname{diag}\left(\lambda_{1}, \ldots, \lambda_{n_{H}-3}\right)+\tilde{Z}^{t} R \tilde{Z} \\
& =m \operatorname{diag}\left(\lambda_{1}, \ldots, \lambda_{n_{H}-3}\right)\left(\tilde{I}+m^{-1} \operatorname{diag}\left(\lambda_{1}^{-1}, \ldots, \lambda_{n_{H}-3}^{-1}\right) \tilde{Z}^{t} R \tilde{Z}\right) .
\end{aligned}
$$

Then,

$$
\left\|\tilde{\Lambda}(m)^{-1}\right\|_{2} \leq \frac{m^{-1} \max _{i \leq n_{H}-3} \lambda_{i}^{-1}}{1-m^{-1} \max _{i \leq n_{H}-3} \lambda_{i}^{-1}\left\|\tilde{Z}{ }^{t} R \tilde{Z}\right\|_{2}},
$$

for sufficiently large $m$, we can conclude the following:

$$
\tilde{\Lambda}(m)^{-1}=\mathcal{O}\left(m^{-1}\right) .
$$

We proceed with the following inversion:

$$
\left[\begin{array}{cc}
\tilde{\Lambda}(m) & \tilde{\delta} \\
\tilde{\delta}^{t} & \eta
\end{array}\right]^{-1}=U(m) V(m) U(m)^{t}
$$

where

$$
\begin{aligned}
U(m) & :=\left[\begin{array}{cc}
\tilde{I} & -\tilde{\Lambda}(m)^{-1} \tilde{\delta} \\
0^{t} & 1
\end{array}\right] \\
V(m) & :=\left[\begin{array}{cc}
\tilde{\Lambda}(m)^{-1} & 0 \\
0^{t} & \left(\eta-\tilde{\delta}^{t} \tilde{\Lambda}(m)^{-1} \tilde{\delta}\right)^{-1}
\end{array}\right]
\end{aligned}
$$

Then, (4.7) implies that

$$
\begin{aligned}
U(m) & =I+\mathcal{O}\left(m^{-1}\right) \\
V(m) & =\left[\begin{array}{cc}
O & 0 \\
0^{t} & \eta^{-1}
\end{array}\right]+\mathcal{O}\left(m^{-1}\right) .
\end{aligned}
$$

Combining the above results, we arrive at

$$
\left[\begin{array}{cc}
\tilde{\Lambda}(m) & \tilde{\delta} \\
\tilde{\delta}^{t} & \eta
\end{array}\right]^{-1}=\left[\begin{array}{cc}
O & 0 \\
0^{t} & \eta^{-1}
\end{array}\right]+\mathcal{O}\left(m^{-1}\right)
$$

and, by (4.6), we have

$$
\begin{aligned}
K_{H H}(m)^{-1} & =Z\left[\begin{array}{cc}
O & 0 \\
0^{t} & \eta^{-1}
\end{array}\right] Z^{t}+\mathcal{O}\left(m^{-1}\right) \\
& =: \quad e_{H} \eta^{-1} e_{H}^{t}+\mathcal{O}\left(m^{-1}\right),
\end{aligned}
$$


which proves (4.1) of the Lemma.

Parts (4.2) and (4.3) follow from simple substitution and using (5.2).

Remark 4.1. If we further decompose DOF associated with $\bar{\Omega}_{H}$ into a set of interior DOF associated with index $I$ and interface DOF with index $\Gamma$, we obtain the following block representation of $K_{H H}$ :

$$
K_{H H}(m)=\left[\begin{array}{ll}
K_{I I}(m) & K_{I \Gamma}(m) \\
K_{\Gamma I}(m) & K_{\Gamma \Gamma}(m)
\end{array}\right] .
$$

The entries in the block $K_{\Gamma \Gamma}(m)$ are assembled from contributions both from finite elements in $\Omega_{H}$ and $\Omega_{L}$, i.e. $K_{\Gamma \Gamma}(m)=A_{\Gamma \Gamma}^{(H)}(m)+A_{\Gamma \Gamma}^{(L)}$.

We further write $e_{H}$ in block form; $e_{H}=\left(e_{I}^{t}, e_{\Gamma}^{t}\right)^{t}$. Finally we note that the off-diagonal blocks have the decomposition:

$$
K_{L H}=\left[\begin{array}{ll}
0 & K_{L \Gamma}
\end{array}\right]=K_{H L}^{t} .
$$

Therefore, the results of Lemma 4.1 can be rewritten as the following:

$$
\begin{aligned}
K_{H H}(m)^{-1} & =e_{H}\left(e_{\Gamma}^{t} K_{\Gamma \Gamma}^{(L)} e_{\Gamma}\right)^{-1} e_{H}^{t}+\mathcal{O}\left(m^{-1}\right), \\
S(m) & =K_{L L}-\left(K_{L \Gamma} e_{\Gamma}\right)\left(e_{\Gamma}^{t} K_{\Gamma \Gamma}^{(L)} e_{\Gamma}\right)^{-1}\left(e_{\Gamma}^{t} K_{\Gamma L}+\mathcal{O}\left(m^{-1}\right),\right. \\
K_{L H} K_{H H}(m)^{-1} & =\left(K_{L \Gamma} e_{\Gamma}\right)\left(e_{\Gamma}^{t} K_{\Gamma \Gamma}^{(L)} e_{\Gamma}\right)^{-1} e_{H}^{t}+\mathcal{O}\left(m^{-1}\right) .
\end{aligned}
$$

\subsection{Qualitative nature of the solution}

We advocate the usage of SPA because it is a very effective tool in gaining qualitative insight about the asymptotic behavior of the solution of the underlying PDE. Through SPA, in Lemma 4.1, we were able to fully reveal the asymptotic behaviour of the submatrices of $K$ in (5.1). This information leads to a characterization of the limit of the underlying discretized inverse operator. We now prove that the solution over the highly-bending island converges to a linear polynomial. In other words, $x_{H}^{\infty} \in \operatorname{span} e_{H}$. This is probably the most fundamental qualitative feature of the solution of the high-contrast biharmonic plate equation.

Lemma 4.2. Let $e_{H}$ as in (3.8). Then,

$$
x_{H}(m)=e_{H} c_{H}+\mathcal{O}\left(m^{-1}\right),
$$

where $c_{H}$ is a $3 \times 1$ vector determined by the solution in the lowly-bending region.

Proof We prove the result by providing an explicit quantification of the limiting process based on Lemma 4.1:

$$
\begin{aligned}
x_{L}(m) & =S^{-1}(m)\left\{b_{L}-K_{L H} K_{H H}^{-1}(m) b_{H}\right\} \\
& =S_{\infty}^{-1}\left\{b_{L}-K_{L H}\left(e_{H} \eta^{-1} e_{H}^{t}\right) b_{H}\right\}+\mathcal{O}\left(m^{-1}\right) \\
& =: x_{L}^{\infty}+\mathcal{O}\left(m^{-1}\right) \\
x_{H}(m) & =K_{H H}^{-1}(m)\left\{b_{H}-K_{H L} x_{L}(m)\right\} \\
= & e_{H} \eta^{-1} e_{H}^{t}\left\{b_{H}-K_{H L} x_{L}^{\infty}\right\}+\mathcal{O}\left(m^{-1}\right) \\
=: & e_{H} c_{H}+\mathcal{O}\left(m^{-1}\right) .
\end{aligned}
$$




\section{CONSTRUCTION OF THE PRECONDITIONER}

The exact inverse of $K$ can be written as:

$$
K^{-1}=\left[\begin{array}{cc}
I_{H H} & -K_{H H}^{-1} K_{H L} \\
0 & I_{L L}
\end{array}\right]\left[\begin{array}{cc}
K_{H H}^{-1} & 0 \\
0 & S^{-1}
\end{array}\right]\left[\begin{array}{cc}
I_{H H} & 0 \\
-K_{L H} K_{H H}^{-1} & I_{L L}
\end{array}\right],
$$

where $I_{H H}$ and $I_{L L}$ denote the identity matrices of the appropriate dimension and the Schur complement $S$ is explicitly given by:

$$
S(m)=K_{L L}-K_{L H} K_{H H}^{-1}(m) K_{H L} .
$$

Let the limit in (4.1) be denoted by $K_{H H}^{\infty \dagger}:=e_{H} \eta^{-1} e_{H}^{t}$. Based on the above perturbation analysis, our proposed preconditioner is defined as follows:

$$
B_{A G K S}(m):=\left[\begin{array}{cc}
I_{H H} & -K_{H H}^{\infty \dagger} K_{H L} \\
0 & I_{L L}
\end{array}\right]\left[\begin{array}{cc}
K_{H H}(m)^{-1} & 0 \\
0 & S_{\infty}^{-1}
\end{array}\right]\left[\begin{array}{cc}
I_{H H} & 0 \\
-K_{L H} K_{H H}^{\infty \dagger} & I_{L L}
\end{array}\right]
$$

We need the following auxillary result to be used in the proof of Theorem 5.1 which characterizes the spectral behaviour of the preconditioned system.

Lemma 5.1. For sufficiently large $m$, we have

$$
K_{H H}^{-1 / 2}=e_{H} \eta^{-1 / 2} e_{H}^{t}+\mathcal{O}\left(m^{-1 / 2}\right),
$$

where $\eta$ is the $3 \times 3$ SPD matrix independent of $m$ defined in (4.4).

Proof We start by writing down the spectral decomposition of $K_{H H}(m)$

$$
Q(m)^{t} K_{H H}(m) Q(m)=\operatorname{diag}\left(\mu_{1}(m), \ldots, \mu_{n_{H}-3}(m), \mu_{n_{H}-2}(m), \mu_{n_{H}-1}(m), \mu_{n_{H}}(m)\right),
$$

where $\left\{\mu_{i}(m): i=1, \ldots, n_{H}\right\}$ denotes a non-increasing ordering of the eigenvalues of $K_{H H}(m)$. Since $K_{H H}(m)$ is SPD, we have $\mu_{i}(m)>0$ for all $i \leq n_{H}$. We use the main fact that eigenvalues and eigenvectors of a symmetric matrix are Lipschitz continuous functions of the matrix entries $[15,30]$.

By (4.5) and (4.8) in Lemma 4.1, we give the following spectral decomposition:

$$
K_{H H}^{-1}(m)=z_{1} 0 z_{1}^{t}+\ldots+z_{n_{H}-3} 0 z_{n_{H}-3}^{t}+e_{H} \eta^{-1} e_{H}^{t}+\mathcal{O}\left(m^{-1}\right) .
$$

Note that $\eta$ in (4.6) is a $3 \times 3$ symmetric, and hence, diagonalizable matrix. We proceed towards a fully diagonalized form of the limiting $K_{H H}^{-1}(m)$. For that, we use the diagonalization of $\eta^{-1}$ :

$$
\eta^{-1}=\hat{z}_{H_{1}} \mu_{H_{1}}^{-1} \hat{z}_{H_{1}}^{t}+\hat{z}_{H_{x}} \mu_{H_{x}}^{-1} \hat{z}_{H_{x}}^{t}+\hat{z}_{H_{y}} \mu_{H_{y}}^{-1} \hat{z}_{H_{y}}^{t} .
$$

Therefore, we have the following expression for the last term in (5.5):

$$
e_{H} \eta^{-1} e_{H}^{t}=\left[z_{H_{1}} z_{H_{x}} z_{H_{y}}\right] \operatorname{diag}\left(\mu_{H_{1}}^{-1}, \mu_{H_{x}}^{-1}, \mu_{H_{y}}^{-1}\right)\left[z_{H_{1}} z_{H_{x}} z_{H_{y}}\right]^{t},
$$

where

$$
\begin{aligned}
{\left[z_{H_{1}} z_{H_{x}} z_{H_{y}}\right] } & :=\left[e_{H_{1}} e_{H_{x}} e_{H_{y}}\right]\left[\hat{z}_{H_{1}} \hat{z}_{H_{x}} \hat{z}_{H_{y}}\right] \\
{\left[e_{H_{1}}, e_{H_{x}}, e_{H_{y}}\right] } & :=e_{H} .
\end{aligned}
$$

2009; 00:1-19 
Now by substituting (5.6) in (5.5), we have the following spectral decomposition which corresponds to the fully diagonalized version:

$$
\begin{aligned}
K_{H H}^{-1}(m) & =z_{1} 0 z_{1}^{t}+\ldots+z_{n_{H}-3} 0 z_{n_{H}-3}^{t}+z_{H_{1}} \mu_{H_{1}} z_{H_{1}}^{t}+z_{H_{x}} \mu_{H_{x}} z_{H_{x}}^{t}+z_{H_{y}} \mu_{H_{y}} z_{H_{y}}^{t}+\mathcal{O}\left(m^{-1}\right) \\
& =: Z_{\infty} \operatorname{diag}\left(0, \ldots, 0, \mu_{H_{1}}^{-1}, \mu_{H_{x}}^{-1}, \mu_{H_{y}}^{-1}\right) Z_{\infty}^{t}+\mathcal{O}\left(m^{-1}\right) .
\end{aligned}
$$

The expression in (5.7) also implies the convergence of the eigenvectors of $K_{H H}(m)$ :

$$
Q(m)=Z_{\infty}+\mathcal{O}\left(m^{-1}\right) .
$$

Note that $Z_{\infty}$ differs from $Z$ in (4.5) only in the last three columns due to diagonalization of $\eta$.

From (5.7), we obtain a characterization of the largest three eigenvalues of $K_{H H}(m)^{-1}$ :

$$
\begin{aligned}
\mu_{n_{H}-2}(m)^{-1} & =\mu_{H_{1}}^{-1}+\mathcal{O}\left(m^{-1}\right) \\
\mu_{n_{H}-1}(m)^{-1} & =\mu_{H_{x}}^{-1}+\mathcal{O}\left(m^{-1}\right) \\
\mu_{n_{H}}(m)^{-1} & =\mu_{H_{y}}^{-1}+\mathcal{O}\left(m^{-1}\right) .
\end{aligned}
$$

Using (5.7) and (5.9), we arrive at the following:

$$
\begin{aligned}
& \operatorname{diag}\left(\mu_{1}(m)^{-1 / 2}, \ldots, \mu_{n_{H}-3}(m)^{-1 / 2}, \mu_{n_{H}-2}(m)^{-1 / 2}, \mu_{n_{H}-1}(m)^{-1 / 2}, \mu_{n_{H}}(m)^{-1 / 2}\right) \\
& =\operatorname{diag}\left(0, \ldots, 0, \mu_{H_{1}}^{-1 / 2}, \mu_{H_{x}}^{-1 / 2}, \mu_{H_{y}}^{-1 / 2}\right)+\mathcal{O}\left(m^{-1 / 2}\right) .
\end{aligned}
$$

By using (5.10) and (5.8), we arrive at the desired result:

$$
\begin{aligned}
K_{H H}(m)^{-1 / 2} & =Q(m) \operatorname{diag}\left(\mu_{1}(m)^{-1 / 2}, \ldots, \mu_{n_{H}}(m)^{-1 / 2}\right) Q(m)^{t} \\
& =Z_{\infty} \operatorname{diag}\left(0, \ldots, 0, \mu_{H_{1}}^{-1 / 2}, \mu_{H_{x}}^{-1 / 2}, \mu_{H_{y}}^{-1 / 2}\right) Z_{\infty}^{t}+\mathcal{O}\left(m^{-1 / 2}\right) \\
& =\left[z_{H_{1}} z_{H_{x}} z_{H_{y}}\right] \operatorname{diag}\left(\mu_{H_{1}}^{-1 / 2}, \mu_{H_{x}}^{-1 / 2}, \mu_{H_{y}}^{-1 / 2}\right)\left[z_{H_{1}} z_{H_{x}} z_{H_{y}}\right]^{t}+\mathcal{O}\left(m^{-1 / 2}\right) \\
& =e_{H} \eta^{-1 / 2} e_{H}^{t}+\mathcal{O}\left(m^{-1 / 2}\right) .
\end{aligned}
$$

The following theorem shows that $B_{A G K S}$ is an effective preconditioner for $m \gg 1$.

Theorem 5.1. For sufficiently large $m$, we have

$$
\sigma\left(B_{A G K S}(m) K(m)\right) \subset\left[1-\mathrm{cm}^{-1 / 2}, 1+\mathrm{cm}^{-1 / 2}\right]
$$

for some constant $c$ independent of $m$, and therefore

$$
\kappa\left(B_{A G K S}(m) K(m)\right)=1+\mathcal{O}\left(m^{-1 / 2}\right) .
$$

Proof Let us factorize the preconditioner as $B_{A G K S}=L^{t} L$ with

$$
L:=\left[\begin{array}{cc}
K_{H H}(m)^{-1 / 2} & 0 \\
-S_{\infty}^{-1 / 2} P_{L H}^{\infty} & S_{\infty}^{-1 / 2}
\end{array}\right],
$$


where the limiting Schur complement $S(m)$ and $K_{L H} K_{H H}^{-1}$ is denoted by $S_{\infty}$ and $P_{L H}^{\infty}$, respectively. We can easily show that

$$
\sigma\left(B_{A G K S} K\right)=\sigma\left(L K L^{t}\right)=\sigma(I+E) .
$$

Note that

$$
P_{L H}^{\infty} K_{H H} P_{L H}^{\infty^{t}}-P_{L H}^{\infty} K_{H L}=K_{L H}\left(e_{H} \eta^{-1} e_{H}^{t} K_{H H} e_{H} \eta^{-1} e_{H}^{t}-e_{H} \eta^{-1} e_{H}^{t}\right) K_{H L}=0 .
$$

We give a step of the operation leading to (5.11). Using (5.12), the $(2,2)$-th block entry of the $L K L^{t}$ reads:

$$
S_{\infty}^{-1 / 2}\left[P_{L H}^{\infty} K_{H H} P_{L H}^{\infty^{t}}-P_{L H}^{\infty} K_{H L}-K_{L H} P_{L H}^{\infty^{t}}+K_{L L}\right] S_{\infty}^{-1 / 2}=I .
$$

The other entries of $L K L^{t}$ can be computed in a similar way.

Using (5.4), we have

$$
E_{L H}=S_{\infty}^{-1 / 2} K_{L H}\left(I_{H H}-e_{H} \eta^{-1} e_{H}^{t} K_{H H}\right) e_{H} \eta^{-1 / 2} e_{H}^{t}+\mathcal{O}\left(m^{-1 / 2}\right)=\mathcal{O}\left(m^{-1 / 2}\right) .
$$

Hence $\rho(E)$, the spectral radius of $E$, is $\mathcal{O}\left(m^{-1 / 2}\right)$, which together with (5.11) completes the proof.

\section{GENERALIZATION TO ELLIPTIC PDES OF ORDER $2 k$}

In essence, the biharmonic plate equation preconditioner is an extension of the construction for the diffusion equation. It is possible to generalize this construction to a family of elliptic PDEs of order $2 k, k>2$. We present how to obtain LRPs from associated bilinear forms. We choose a different perspective than the one in Section 3. We start with a canonical bilinear form and show the modification it needs to go through in order to construct LRPs.

Let the generalized problem be stated as follows: Find $u \in H_{0}^{k}(\Omega)$ such that

$$
T_{k} u:=(-1)^{k} \nabla^{k}\left(\alpha_{k} \nabla^{k} u\right)=f \quad \text { in } \Omega .
$$

The straightforward bilinear form associated to (6.1) is obtained by application of Green's formula $k$ times:

$$
\int_{\Omega} \nabla^{k}\left(\alpha_{k} \nabla^{k} u\right) v d x=\int_{\Omega} \alpha_{k} \nabla^{k} u \nabla^{k} v d x+\text { boundary terms. }
$$

Then, we define a bilinear form corresponding to (6.1) which can be seen as a generalization of the canonical bilinear form in (2.5):

$$
\tilde{a}_{k}(u, v):=\int_{\Omega} \alpha_{k} \nabla^{k} u \nabla^{k} v d x .
$$

Without modification, $\tilde{a}_{k}(\cdot, \cdot)$ cannot lead to LRPs because $\tilde{a}_{k}(v, v)$ is not $H_{0}^{k}(\Omega)$-coercive. This is due to the fact that $\tilde{a}_{k}(v, v)=0$ for $v \in \mathcal{P}_{k-1} \cap H_{0}^{k}(\Omega)$. Hence, the stiffness matrix induced by (6.3) has a large kernel involving elements from $\mathcal{P}_{k-1}^{h} \cap V^{h}$ which indicates that extraction of a Neumann matrix with a low-dimensional kernel is impossible. In order to overcome this complication, we utilize a modified bilinear form:

$$
a_{k}(u, v)=\tilde{a}_{k}(u, v)+\left(1-\sigma_{k}\right) \hat{a}_{k}(u, v) .
$$

The bilinear form should maintain the following essential properties:

2009; 00:1-19 
1. $H_{0}^{k}(\Omega)$-coercive.

2. $V_{\mathcal{P}_{k-1}(\Omega)}$-coercive.

3. Corresponds to a strong formulation giving $T_{k} u$ in (6.1) precisely,

where $V_{\mathcal{P}_{k-1}(\Omega)}$ is a closed subspace such that $V_{\mathcal{P}_{k-1}}(\Omega) \cap \mathcal{P}_{k-1}=\emptyset$ and $\mathcal{P}_{k-1}$ denotes the set of polynomials of degree at most $k-1$.

The above properties (1) and (2) will be immediately satisfied if the generalization of (3.5) holds for the modified bilinear form:

$$
a_{k}(v, v) \geq c_{k}|v|_{H^{k}(\Omega)}^{2} .
$$

A similar construction of the Neumann matrix can be immediately generalized as follows:

$$
\left\langle\mathcal{N}_{H H}^{(k)} \underline{\phi}, \underline{\psi}\right\rangle:=a_{k}\left(\phi_{H}^{h}, \psi_{H}^{h}\right) .
$$

The low-rank perturbations arise from the following decomposition of $K_{H H}^{(k)}(m)$ :

$$
K_{H H}^{(k)}(m)=m \mathcal{N}_{H H}^{(k)}+R^{(k)}, \quad\left(K_{H H}^{(k)}(m)\right)^{-1}=e_{H}^{(k)} \eta^{(k)^{-1}} e_{H}^{(k)^{t}}+\mathcal{O}\left(m^{-1}\right),
$$

where $\eta^{(k)}:=e_{H}^{(k)^{t}} K_{H H}^{(k)} e_{H}^{(k)}$. LRP is produced by $e_{H}^{(k)} \in \mathcal{P}_{k-1}^{h}$ because the rank is equal to the cardinality of the basis polynomials in $\mathcal{P}_{k-1}^{h}$.

$$
\operatorname{ker} \mathcal{N}_{H H}^{(k)}=\left.\mathcal{P}_{k-1}^{h}\right|_{\bar{\Omega}_{H}} .
$$

Due to $(2.6), a_{2}(\cdot, \cdot)$ in (2.3) corresponds to the strong formulation $T_{2}$ exactly. Let us denote the strong formulation to which $a_{k}(\cdot, \cdot)$ corresponds by $\hat{T}_{k}$. We have $\hat{T}_{k}=T_{k}, k=1,2$ for the high-contrast diffusion and biharmonic plate equations, respectively:

$$
\begin{aligned}
& a_{1}(v, v):=\left(\nabla v, \alpha_{1} \nabla v\right) \\
& a_{2}(v, v):=\sigma_{2}\left(\nabla^{2} v, \alpha_{2} \nabla^{2} v\right)+\alpha_{2}\left(1-\sigma_{2}\right)|v|_{H^{2}(\Omega)}^{2}
\end{aligned}
$$

However, for general $k, a_{k}(\cdot, \cdot)$ may not correspond to $T_{k}$. In addition, one may need more general boundary conditions if similar zero contributions in (2.6) can be obtained for general $k$. Further research is needed to see if such boundary conditions are physical. Currently, it is also unclear for which applications such general PDEs can be used. However, there are interesting invariance theory implications when one employs bilinear forms corresponding to rotationally invariant functions compatible to energy definition in (2.2). This allows a generalization of the energy notion and may be the subject for future research. For further information, we list the relevant bilinear forms that are composed of rotationally invariant functions derived by the utilization of invariance theory.

$$
\begin{aligned}
& a_{3}(v, v):=\sigma_{3}\left(\nabla^{3} v, \alpha_{3} \nabla^{3} v\right)+\alpha_{3}\left(1-\sigma_{3}\right)|v|_{H^{3}(\Omega)}^{2} \\
& a_{4}(v, v):=\sigma_{4}\left(\nabla^{4} v, \alpha_{4} \nabla^{4} v\right)+\alpha_{4}\left(1-\sigma_{4}\right)|v|_{H^{4}(\Omega)}^{2}+\alpha_{4} \gamma_{4}\left|\nabla^{2} v\right|_{H^{2}(\Omega)}^{2} .
\end{aligned}
$$

Note that the above bilinear forms satisfy (6.4). 


\section{NUMERICAL EXPERIMENTS}

The goal of the numerical experiments is to compare the performance of the two preconditioners: AGKS and MG. The domain is a unit square whose coarsest level triangulation consists of 32 triangles. We consider the case of a single highly-bending island located at the region $[1 / 4,2 / 4] \times[1 / 4,2 / 4]$ consisting of 2 coarsest level triangles. For an extension to the case of multiple disconnected islands, one can refer to [4, Sections 3 and 4]. The implementation of HCT and Morley discretizations are based on Pozrikidis' software provided in [28]. The problems sizes of HCT and Morley discretizations are 131, 451, 1667, 6403 and 81, 289, 1089, 4225 for levels $1,2,3$ and 4 , respectively.

We denote the norm of the relative residual at iteration $i$ by $r r^{(i)}$ :

$$
r r^{(i)}:=\frac{\left\|r^{(i)}\right\|_{2}}{\left\|r^{(0)}\right\|_{2}}
$$

where $r^{(i)}$ denotes the residual at iteration $i$ with a stopping criterion of $r r^{(i)} \leq 10^{-7}$. In Tables I-VIII, preconditioned conjugate gradient iteration count and the average reduction factor are reported for combinations of preconditioner, smoother types, and number of smoothing iterations. The average reduction factor of the residual is defined as:

$$
\left(r r^{(i)}\right)^{1 / i}
$$

We enforce an iteration bound of 60 . If the method seems to converge slightly beyond this bound, we denote it by $60^{+}$, whereas, stalling is denoted by $\infty$.

We use Galerkin variational approach to construct the coarser level algebraic systems. The multigrid preconditioner MG is derived from the implementation by Aksoylu, Bond, and Holst [3]. We employ a V(1,1)-cycle with point symmetric Gauss-Seidel (sGS) and point GaussSeidel (GS) smoothers. A direct solver is used for the coarsest level.

By exploiting the fact that $S_{\infty}$ in (1.1) is only a LRP of $K_{L L}$, we can build robust preconditioners for $S_{\infty}$ in (5.3) via standard multigrid preconditioners. (1.1) implies that

$$
S_{\infty}=K_{L L}-v \eta^{-1} v^{T},
$$

where $v:=K_{L H} e_{H}$. If $M_{L L}$ denotes a standard multigrid V-cycle for $K_{L L}$, we can construct an efficient and robust preconditioner $\tilde{S}^{-1}$ for $S_{\infty}$ using the Sherman-Morrison-Woodbury formula, i.e.

$$
\tilde{S}^{-1}:=M_{L L}+M_{L L} v\left(\eta-v^{T} M_{L L} v\right)^{-1} v^{T} M_{L L} .
$$

Note also that we can precompute and store $M_{L L} v$ during the setup phase. This means that we only need to apply the multigrid V-cycle $M_{L L}$ once per iteration. Therefore, the following practical version of preconditioner (5.3) is used in the implementation:

$$
\tilde{B}_{A G K S}:=\left[\begin{array}{cc}
I_{H H} & -K_{H H}^{\infty} K_{H L} \\
0 & I_{L L}
\end{array}\right]\left[\begin{array}{cc}
M_{H H} & 0 \\
0 & \tilde{S}^{-1}
\end{array}\right]\left[\begin{array}{cc}
I_{H H} & 0 \\
-K_{L H} K_{H H}^{\infty} & I_{L L}
\end{array}\right] .
$$

We construct two different multilevel hierarchies for multigrid preconditioners $M_{H H}$ in (7.2) and $M_{L L}$ in (7.1) for DOF corresponding to $\Omega_{H}$ and $\Omega_{L}$, respectively. For prolongation, linear 
Table I. AGKS + HCT + sGS + smooth number 1-5-10

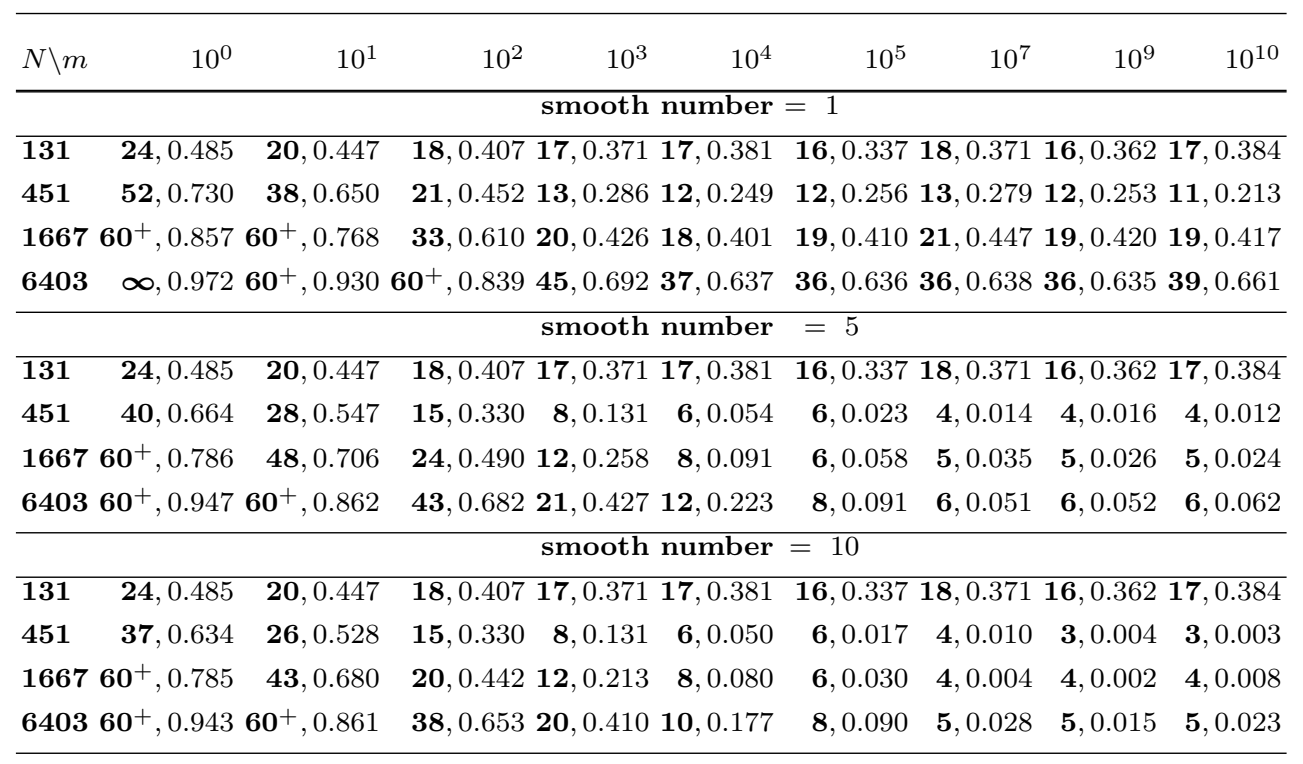

Table II. AGKS + HCT + GS + smooth number 1-5-10

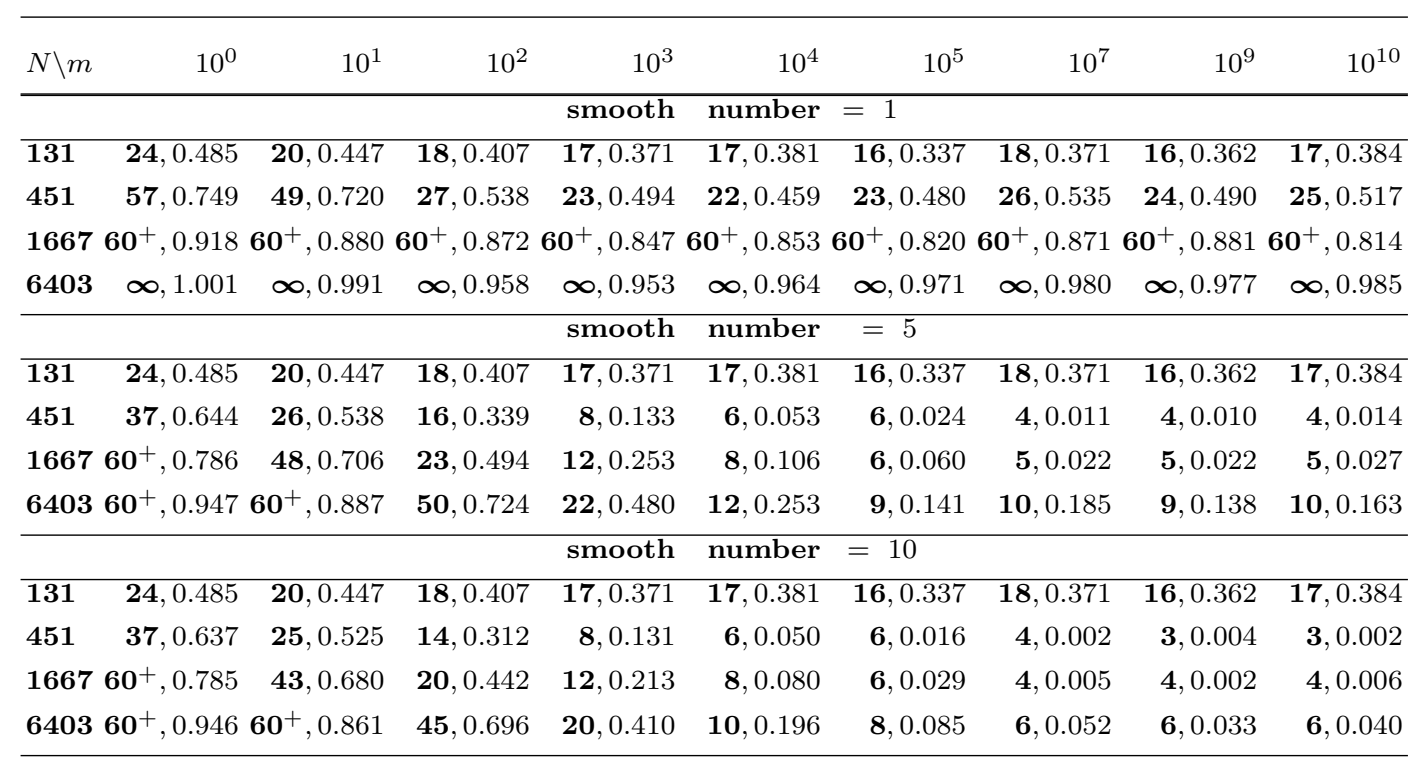


Table III. AGKS + Morley + sGS + smooth number 1-5-10

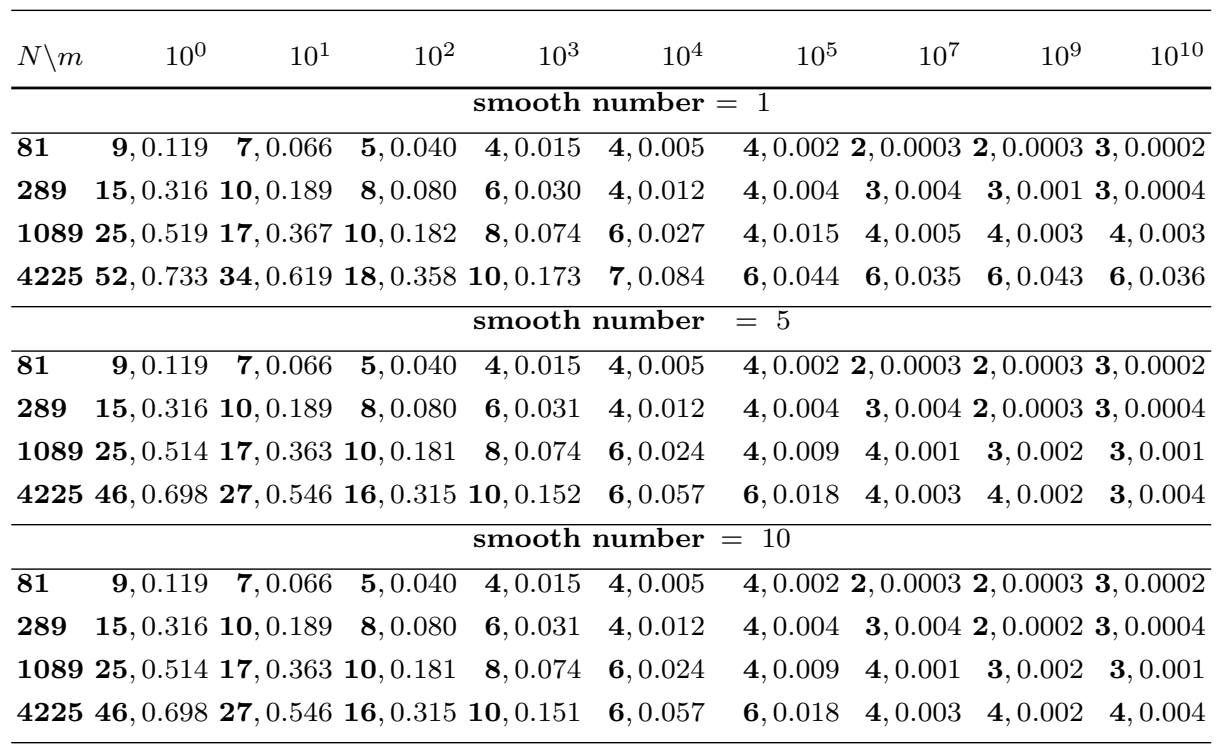

Table IV. AGKS + Morley + GS + smooth number 1-5-10

\begin{tabular}{|c|c|c|c|c|c|c|c|c|c|}
\hline$m$ & $10^{0}$ & $10^{1}$ & $10^{2}$ & 3 & $10^{4}$ & 10 & $10^{7}$ & $10^{9}$ & 10. \\
\hline \multicolumn{10}{|c|}{ smooth number $=1$} \\
\hline 81 & 0.119 & 0.066 & $\mathbf{5}, 0.040$ & $4,0.015$ & $4,0.005$ & $4,0.002$ & $\mathbf{2}, 0.0003$ & $\mathbf{2}, 0.0003$ & $\mathbf{3}, 0.0002$ \\
\hline 289 & $\mathbf{1 5}, 0.329$ & $\mathbf{1 0}, 0.189$ & $8,0.080$ & $6,0.031$ & $\mathbf{4}, 0.012$ & $4,0.006$ & $3,0.005$ & $3,0.003$ & $3,0.003$ \\
\hline 089 & $\mathbf{2 8}, 0.550$ & $\mathbf{1 9}, 0.402$ & $\mathbf{1 0}, 0.192$ & $\mathbf{8}, 0.085$ & $6,0.043$ & $\mathbf{5}, 0.040$ & $\mathbf{5}, 0.037$ & $6,0.030$ & $5,0.039$ \\
\hline \multirow[t]{2}{*}{4225} & $\mathbf{5 9}, 0.760$ & $\mathbf{3 8}, 0.651$ & $\mathbf{2 0}, 0.433$ & $\mathbf{1 2}, 0.249$ & $\mathbf{1 1}, 0.204$ & $\mathbf{1 1}, 0.214$ & $\mathbf{1 1}, 0.208$ & $\mathbf{1 1}, 0.210$ & $\mathbf{1 1}, 0.206$ \\
\hline & & & & smooth & number & $=5$ & & & \\
\hline & $\mathbf{9}, 0.119$ & 0.066 & $\mathbf{5}, 0.040$ & $4,0.015$ & $4,0.005$ & $4,0.002$ & $\mathbf{2 ,}, 0.0003$ & 2,0 & $\mathbf{3}$, \\
\hline 39 & $\mathbf{1 5}, 0.316$ & $\mathbf{1 0}, 0.189$ & $\mathbf{8}, 0.080$ & $6,0.031$ & $4,0.012$ & $4,0.004$ & $\mathbf{3}, 0.004$ & $\mathbf{2}, 0.0002$ & $\mathbf{3}, 0.0004$ \\
\hline 1089 & $\mathbf{2 5}, 0.514$ & $\mathbf{1 7}, 0.363$ & $\mathbf{1 0}, 0.181$ & $8,0.074$ & $6,0.024$ & $4,0.009$ & $4,0.001$ & $3,0.002$ & $3,0.001$ \\
\hline \multirow[t]{2}{*}{4225} & $\mathbf{4 6}, 0.698$ & $\mathbf{2 7}, 0.0546$ & 16,0 & $\mathbf{1 0}, 0.152$ & $6,0.057$ & $6,0.018$ & $4,0.003$ & $\mathbf{4}, 0.002$ & $3,0.004$ \\
\hline & & & & smooth & umber & $=10$ & & & \\
\hline & 0.119 & 0.066 & $\mathbf{5}, 0.040$ & $4,0.015$ & $4,0.005$ & $4,0.002$ & $\mathbf{2}, 0.0003$ & $\mathbf{2}, 0.0003$ & 002 \\
\hline 289 & $\mathbf{1 5}, 0.316$ & $\mathbf{1 0}, 0.189$ & $\mathbf{8}, 0.080$ & $6,0.031$ & $4,0.012$ & $4,0.004$ & $\mathbf{3}, 0.004$ & $\mathbf{2}, 0.0001$ & $\mathbf{3}, 0.0004$ \\
\hline 1089 & $\mathbf{2 5}, 0.514$ & $\mathbf{1 7}, 0.363$ & $\mathbf{1 0}, 0.181$ & $8,0.074$ & $6,0.024$ & 4, 0.009 & $4,0.001$ & 3, 0.002 & $3,0.001$ \\
\hline 4225 & $46,0.698$ & $\mathbf{2 7}, 0.0546$ & $\mathbf{1 6}, 0.315$ & $\mathbf{1 0}, 0.152$ & $6,0.057$ & $6,0.018$ & $4,0.003$ & $4,0.002$ & 3, 0.003 \\
\hline
\end{tabular}


Table V. MG + HCT + sGS + smooth number 1-5-10

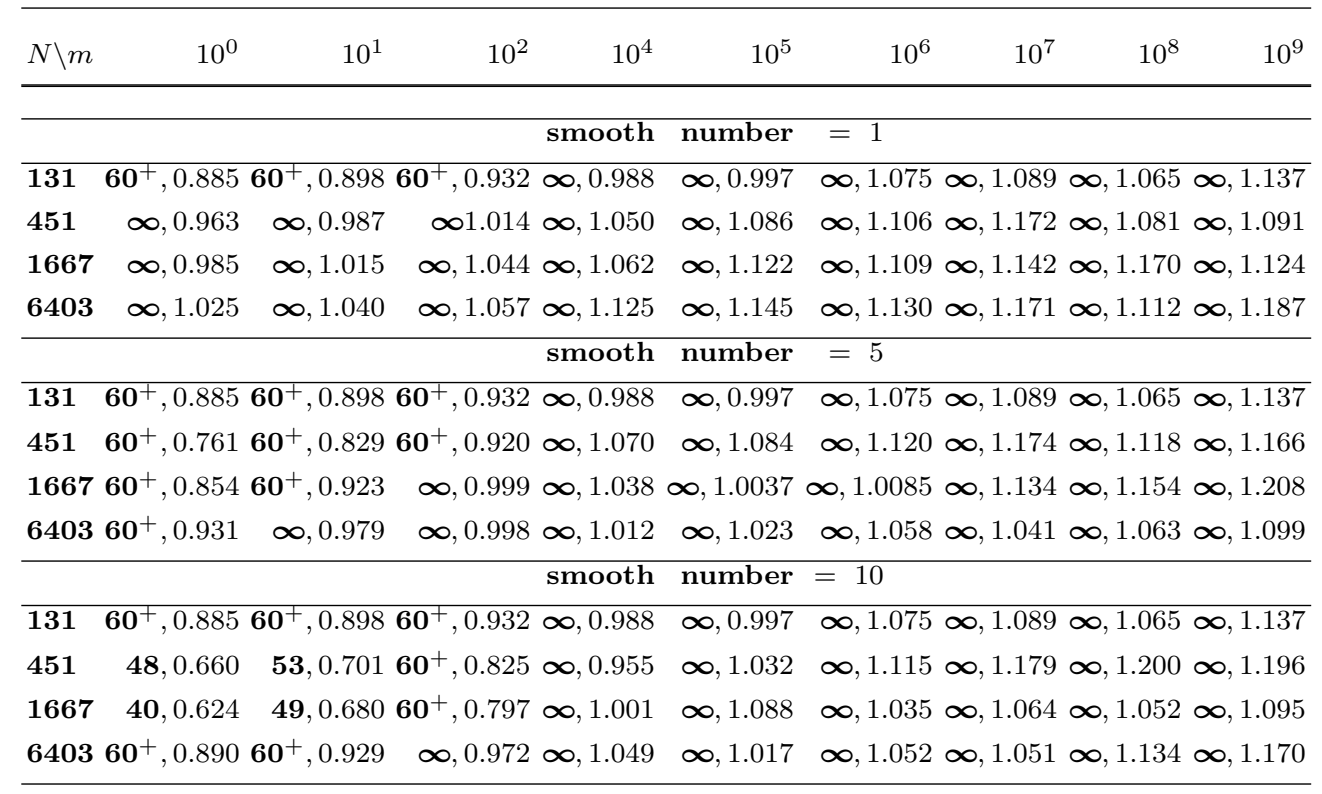

Table VI. MG + HCT + GS + smooth number 1-5-10

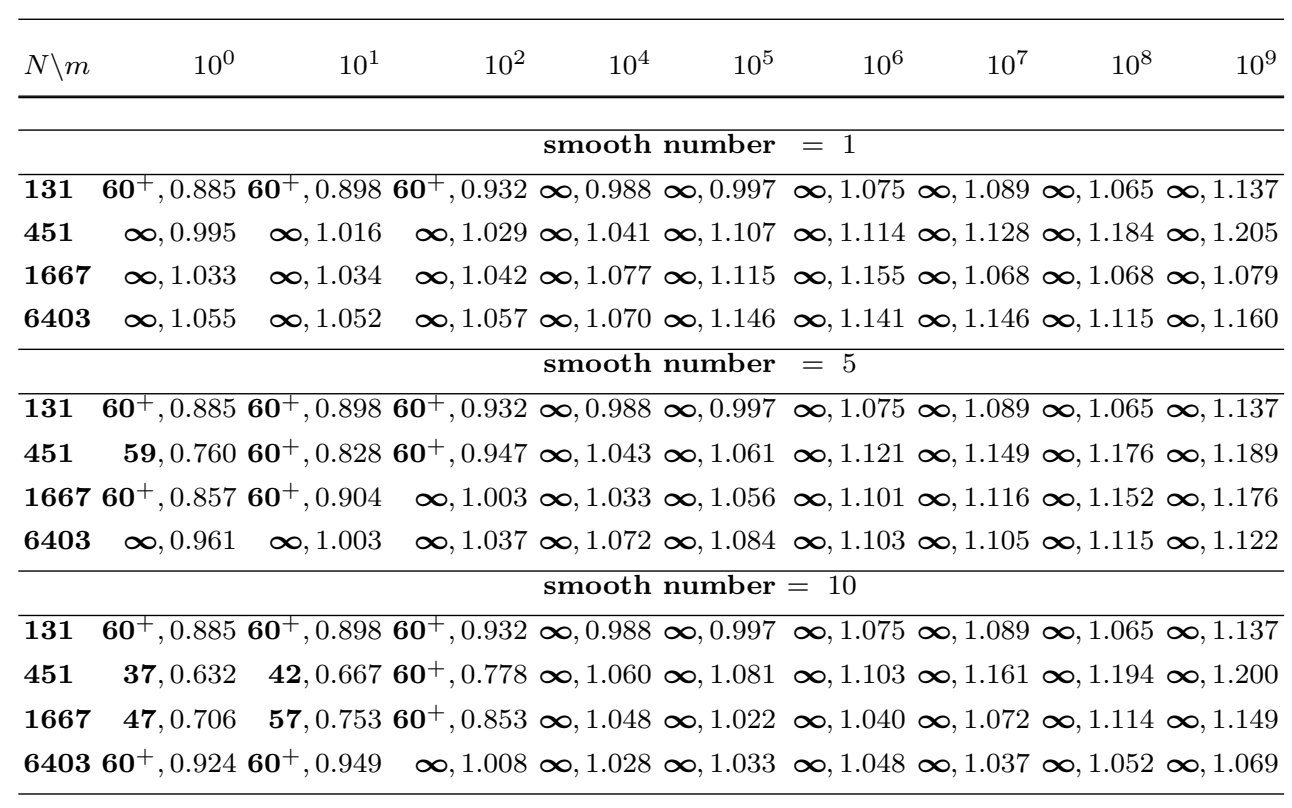


Table VII. MG + Morley + sGS + smooth number 1-5-10

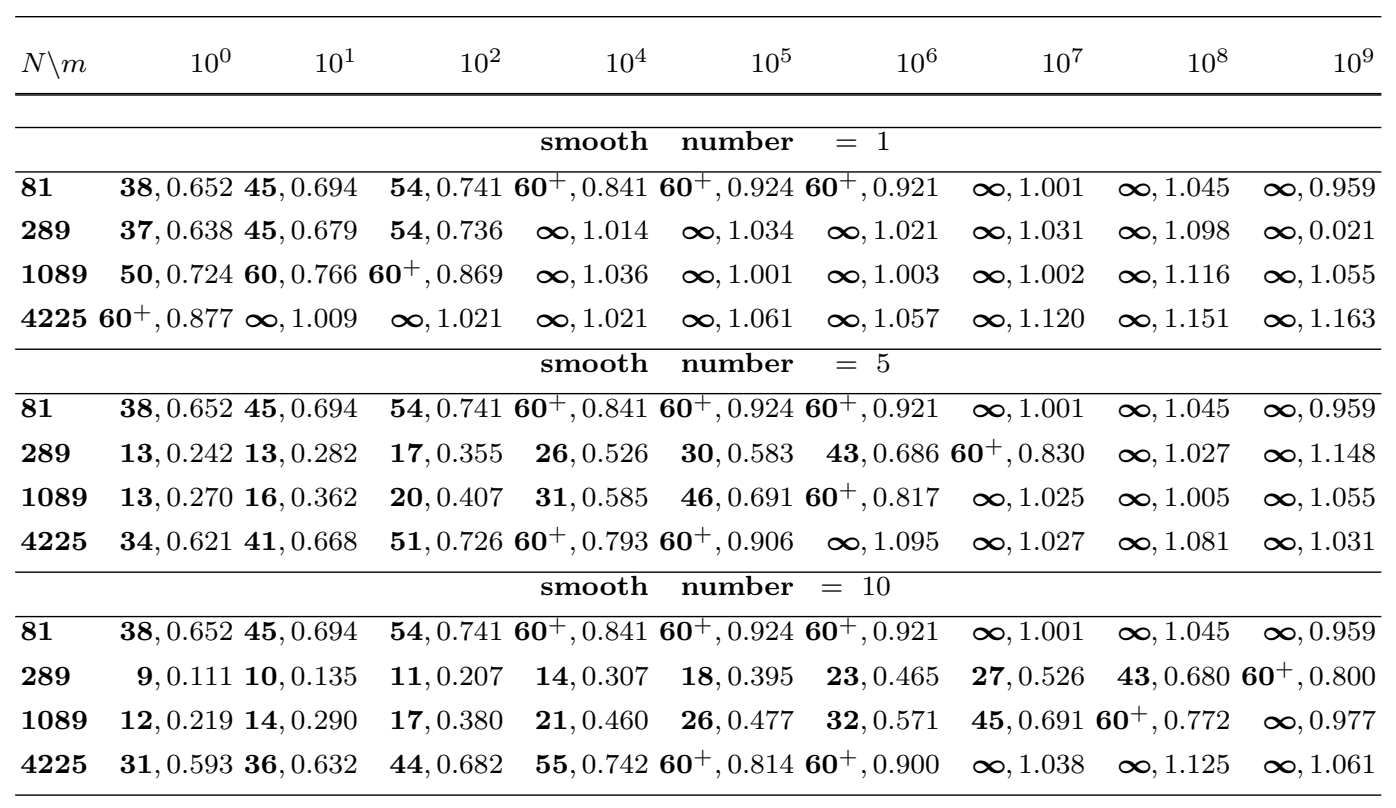

Table VIII. MG + Morley + GS + smooth number 1-5-10

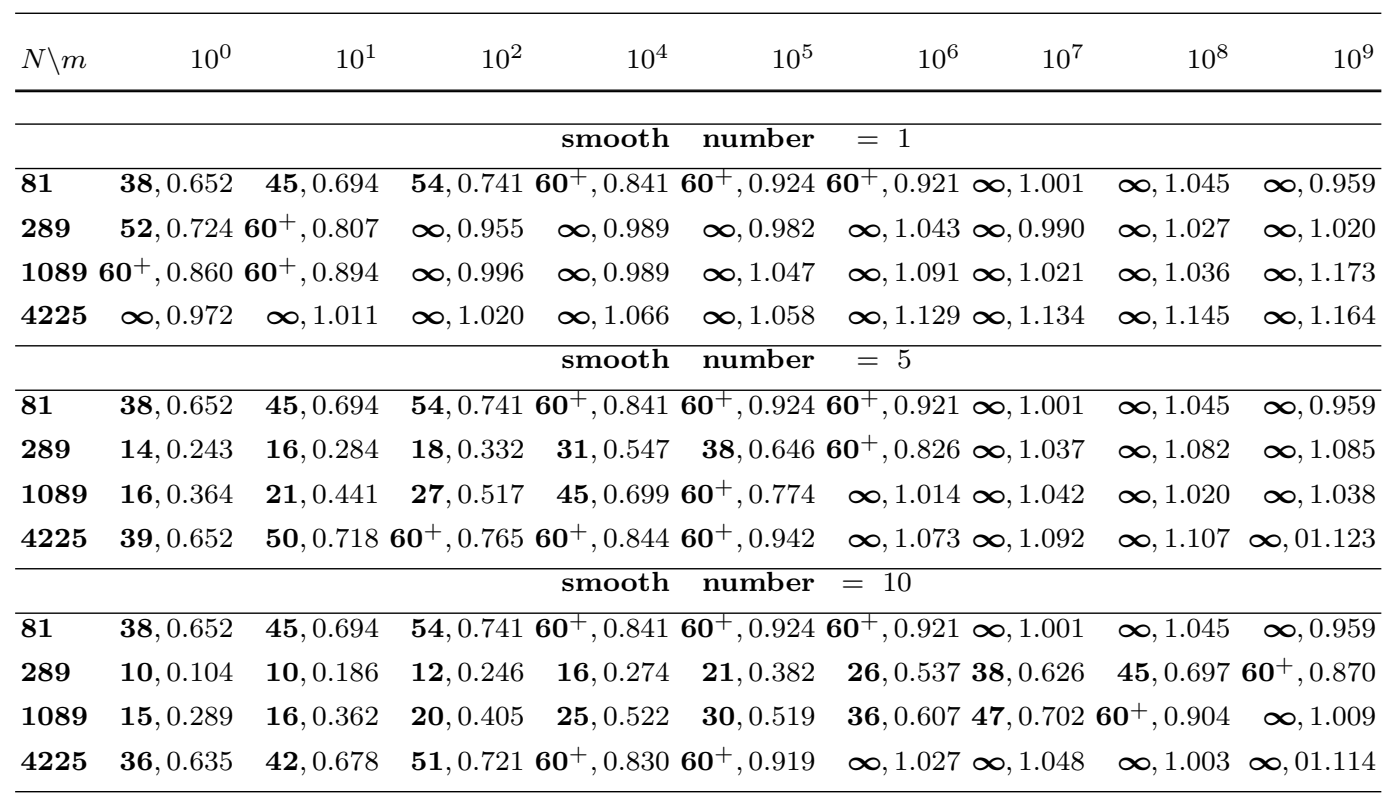


interpolation is used as in [8]. The prolongation matrices $P_{H H}$ and $P_{L L}$ are extracted from the prolongation matrix for whole domain $\Omega$ in the fashion following (3.3):

$$
P=\left[\begin{array}{ll}
P_{H H} & P_{H L} \\
P_{L H} & P_{L L}
\end{array}\right] \text {. }
$$

As emphasized in our preceding paper [4], AGKS can be used purely as an algebraic preconditioner. Therefore, the standard multigrid preconditioner constraint that the coarsest level mesh resolves the boundary of the island is automatically eliminated. However, for a fair comparison, we enforce the coarsest level mesh to have that property.

We do not observe convergence improvement when a subdomain deflation strategy based on the smallest eigenvalues is used as in the diffusion equation case [6]. The eigenvectors of the Neumann matrix, $e_{H}$ in (3.8), cannot approximate the eigenvectors corresponding to the smallest eigenvalues of $K_{H H}$ which are of $\mathcal{O}(1)$ (see Figure 2) since the remainder matrix $R$ in (3.7) is of $\mathcal{O}\left(10^{4}\right)$. Therefore, a deflation strategy utilizing $e_{H}$ will not necessarily guarantee deflation of the smallest eigenvalues of $K_{H H}$ in the biharmonic case.

We first observe that the Morley discretization provides faster convergence for both preconditioners. Then, we have the following results regarding the effect of number of smoothing iterations on the convergence behaviour. The convergence of MG heavily depends on the number of smoothing iterations, i.e., the more the smoothing iteration, the faster the convergence. For the HCT discretization, AGKS requires more than a single smoothing iteration for convergence; see Tables I and II. However, for the Morley discretization, even with the same minimal number of smoothing iteration, AGKS leads to convergence; see Tables III and IV. The choice of 5 smoothing iterations is sufficient for AGKS to reach $h$-robustness and its peak performance. Hence, we can conclude that AGKS clearly enjoys $h$-robustness. In contrast, $\mathrm{MG}$ is not $h$-robust regardless of the $m$ value and the smoothing number; see Tables V, VI, VII, and VIII. MG is totally ineffective as the problem size increases for both discretizations, and more obviously for HCT.

Finally, we report the $m$-robustness results. The loss of $m$-robustness of MG can be observed consistently for all $m$ values; see Tables V, VI, VII, and VIII. The AGKS preconditioner becomes more effective with increasing $m$ and reaches its peak performance by maintaining an optimal iteration count for all $m \geq 10^{5}$. This indicates that $m \geq 10^{5}$ corresponds to the asymptotic regime. Even increasing the $m$ value from $10^{2}$ to $10^{3}$ reduces the iteration count significantly, a clear sign of close proximity to the asymptotic regime. In addition, the AGKS outperforms MG even for $m=1$. Consequently, for both discretizations, we infer that AGKS is $m$-robust.

\section{REFERENCES}

1. B. Aksoylu And H. R. Beyer, Results on the diffusion equation with rough coefficients. Submitted to SIAM J. Math. Anal., 2008.

2. - On the characterization of the asymptotic cases of the diffusion equation with rough coefficients and applications to preconditioning, Numer. Funct. Anal. Optim., 30 (2009), pp. 405-420.

3. B. Aksoylu, S. Bond, And M. Holst, An Odyssey into Local Refinement and Multilevel Preconditioning III: Implementation and Numerical Experiments, SIAM J. Sci. Comput., 25 (2003), pp. 478-498.

4. B. Aksoylu, I. G. Graham, H. Klie, and R. Scheichl, Towards a rigorously justified algebraic preconditioner for high-contrast diffusion problems, Comput. Vis. Sci., 11 (2008), pp. 319-331. doi:10.1007/s00791-008-0105-1.

2009; 00:1-19 
5. B. AKsoylu And H. Klie, A family of physics-based preconditioners for solving elliptic equations on highly heterogeneous media, Appl. Num. Math., 59 (2009), pp. 1159-1186. doi:10.1016/j.apnum.2008.06.002.

6. B. AKSOYLu AND Z. YeTER, Robust multigrid preconditioners for cell-centered finite volume discretization of the high-contrast diffusion equation. Submitted to Comput. Vis. Sci., 2009.

7. N. S. Bakhvalov And A. V. KnYazev, A new iterative algorithm for solving problems of the fictitious flow method for elliptic equations, Soviet Math. Dokl., 41 (1990), pp. 481-485.

8. D. Braess And P. Peisker, A conjugate gradient method and a multigrid algorithm for Morley's finite element approximation of the biharmonic equation, Numerische Mathematik, 50 (1987), pp. 567-586.

9. P. G. CiARLEt, The Finite Element Method for Elliptic Problems, Classics in applied mathematics, SIAM, Philadelphia, PA, 2002.

10. R. W. Clough and J. L. Tocher, Finite element stiffness matrices for analysis of plates in bending, in Proceedings of the conference on matrix methods in structural mechanics, 1965.

11. Q. A. DANG, Iterative method for solving the Neumann boundary value problem for biharmonic type equation, J. Comput. Appl. Math, 196 (2006), pp. 643-643.

12. I. G. Graham And M. J. HagGer, Unstructured additive Schwarz-conjugate gradient method for elliptic problems with highly discontinuous coefficients, SIAM J. Sci. Comp., 20 (1999), pp. 2041-2066.

13. R. O. Grossi, On the existence of weak solutions in the study of anisotropic plates, J. Sound Vibration, 242 (2001), pp. 542-552.

14. M. R. HANISCH, Multigrid preconditioning for the biharmonic Dirichlet problem, SIAM J. Numer. Anal., 30 (1993), pp. 184-214.

15. T. KAто, A short introduction to perturbation theory for linear operators, Springer, Berlin, Germany, 1982.

16. A. KNYAZEv And O. Widlund, Lavrentiev regularization + Ritz approximation = uniform finite element error estimates for differential equations with rough coefficients, Math. Comp., 72 (2003), pp. 17-40.

17. J. Maes And A. Bultheel, A hierarchical basis preconditioner for the biharmonic equation on the sphere, IMA J. Numer. Anal., 26 (2006), pp. 563-583.

18. G. D. Manolis, T. V. Rangelov, and R. P. Shaw, The non-homogeneous biharmonic plate equation: fundamental solutions, Internat. J. Solids Structures, 40 (2003), pp. 5753-5767.

19. L. Marcinkowski, An additive Schwarz method for mortar finite element discretizations of the 4 th order elliptic problem in 2D, Electron. Trans. Numer. Anal., 26 (2007), pp. 34-54.

20. A. MAYO, The fast solution of Poisson's and the biharmonic equations on irregular regions, SIAM J. Numer. Anal., 21 (1984), pp. 285-299.

21. A. Mayo And A. Greenbaum, Fast parallel iterative solution of Poisson's and the biharmonic equations on irregular regions, SIAM J. Sci. Statist. Comput., 13 (1992), pp. 101-118.

22. M. Mihajlovic And D. Silvester, A black-box multigrid preconditioner for the biharmonic equation, BIT, 44 (2004), pp. 151-163.

23. M. Minajlović And D. Silvester, Efficient parallel solvers for the biharmonic equation, Parallel Computing, 30 (2004), pp. 35-55.

24. K. L. Miller and C. O. Horgan, End effects for plane deformations of an elastic anisotropic semiinfinite strip, J. Elasticity, 38 (1995), pp. 261-316.

25. L. S. D. Morley, The triangular equilibrium problem in the solution for plate bending problems, Aero. Quart., 19 (1968), pp. 149-169.

26. P. Oswald, Hierarchical conforming finite element methods for the biharmonic equation, SIAM J. Numerical Analysis, 29 (1992), pp. 1610-1625.

27. — Multilevel preconditioners for discretizations of the biharmonic equation by rectangular finite elements, Numer. Lin. Alg. Appl., 2 (1995), pp. 487-505.

28. C. PozRikidis, Introduction to Finite and Spectral Element Methods using MATLAB, Chapman \& Hall/CRC, Boca Raton, FL, 2005.

29. T. S. WANG, A Hermite cubic immersed finite element space for beam design problems. Master thesis, Department of Mathematics, Virginia Polytechnic Institute and State University, 2005.

30. D. S. WAtKins, Fundamentals of Matrix Computations, Wiley-Interscience; second edition, New York, 2002 .

31. X. Zhang, Multilevel Schwarz methods for the biharmonic Dirichlet problem, SIAM J. Sci. Comput., 15 (1994), pp. 621-644. 